\title{
Vivienda social en la periferia de la Ciudad de México como mecanismo de acumulación de capital y control social
}

\author{
Social housing in the periphery of Mexico City as a mechanism for capital accumulation and social control
}

\section{Luis Alberto Salinas Arreortua*}

Recibido: 23 de enero de 2019

Aceptado: 09 de mayo de 2019

\section{Resumen}

La gubernamentabilidad puede ser entendida como la creación de instituciones, estrategias e instrumentos jurídicos y políticos a través de los cuales se ejerce el poder. El objetivo del presente artículo es develar aspectos sobre la acumulación de capital y control social a partir de la construcción de vivienda social en la periferia de la Ciudad de México. La implementación de un sistema de crédito otorgado por los organismos nacionales de vivienda para la compra de vivienda, la generación del anhelo del acceso a la vivienda en propiedad, son mecanismos que han repercutido en la reproducción y acumulación de capital, mientras que, la generación de la deuda y el confinamiento de sectores de población de bajos ingresos en la periferia urbana, forman parte de un mecanismo de control de la sociedad. A partir de una revisión sobre el concepto de gubernamentabilidad y mediante el uso de datos sobre los créditos otorgados por instituciones públicas y entidades financieras, se propone una discusión sobre la política de vivienda como un mecanismo que ha servido para la acumulación de capital y el control social.

Palabras clave: biopolítica, control social, gubernamentabilidad, política de vivienda, reproducción de capital.

\begin{abstract}
Governmentability can be understood as the creation of institutions, strategies and legal and political instruments through which power is exercised. The objective of this article is to reveal aspects of capital accumulation and social control based on the construction of social housing in the outskirts of Mexico City. The implementation of a credit system granted by the national housing agencies for the purchase of homes, the generation of the yearning for access to home ownership, are mechanisms that have had an impact on the reproduction and accumulation of capital, while the generation of debt and the confinement of sectors of low-income population in the urban periphery, are part of a mechanism of control of society. Based on a review of the concept of governability and through the use of data on credits granted by public institutions and financial entities, a discussion on housing policies is proposed as mechanism that served to accumulate capital and social control.
\end{abstract}

Keywords: biopolitics, control of society, housing policy, Governmentability, reproduction of capital.

* Filiación: Instituto de Geografía, Universidad Nacional Autónoma de México, Ciudad de México, México. Contacto: Isalinas@igg.unam.mx

Este trabajo es resultado del proyecto de investigación titulado Política de vivienda y gestión urbana. El capital inmobiliario en los procesos de gentrificación en la Ciudad de México, financiado por el Programa de Apoyo a Proyectos de Investigación e Innovación Tecnológica (PAPIIT- IA300318) de la Universidad Nacional Autónoma de México, desarrollado en el Instituto de Geografía.

Cómo citar: Salinas Arreortua, L.A. (2019). Vivienda social en la periferia de la Ciudad de México como mecanismo de acumulación de capital y control social. Revista de Urbanismo, 40, 1-13. https://doi.org/10.5354/0717-5051.2018.52243 


\section{Introducción}

En el análisis sobre el proceso de conocimiento, Karel Kosik (1967) argumenta que una forma para aproximarse a las explicaciones de los procesos que se tratan en ciencias sociales es a partir de una relación dialéctica del fenómeno a la esencia. La apariencia (fenómeno) de los procesos son sus propiedades y relaciones que le dan sentido, que apreciamos de manera evidente, en la cual se revela, de forma parcial, la esencia. El contenido (esencia) constituye los elementos y relaciones más profundas que explican el proceso. No obstante, la manera en la cual percibimos los procesos estará influenciada por aspectos históricos, culturales, institucionales, políticos y económicos.

La política de vivienda, como fenómeno, se le ha analizado desde el ámbito económico y político, sin embargo, poco se ha discutido como un mecanismo de control hacia los sujetos y la sociedad. En la Ciudad de México ${ }^{1}$, la expansión en las dos últimas décadas se ha caracterizado por la construcción de vivienda dirigida para población con empleo formal y con bajos ingresos, de esta manera, se excluye a amplios sectores de la población. Las características de esta expansión urbana, está conformando una ciudad con una concentración de población de bajos ingresos en la periferia urbana. Es una manera de confinar a población de escasos recursos económicos en la periferia urbana.

Más allá de proponer un análisis sobre la esencia, se pretende discutir la política de vivienda como un mecanismo de acumulación de capital y control de la sociedad, es decir, concebirla en términos de lo que Foucault llama gubernamentabilidad entendida como el conjunto de mecanismos y estrategias construidas por instituciones, procedimientos y tácticas que permiten ejercer el poder (Foucault, 2014). El objetivo del presente artículo es develar aspectos sobre la acumulación de capital y control de la sociedad contenidos y ejercidos mediante la política de vivienda social. De manera específica, se discute el sistema de crédito público (para adquisición de vivienda) y el aspecto cultural de acceder a una vivienda en propiedad, como estrategias que han permitido la reproducción y acumulación de capital. Por otra parte, esta forma de consumo en propiedad a través de la generación de la deuda y el confinamiento de sectores de población de bajos ingresos en la periferia urbana, forman parte de un mecanismo de control social. Es pensar la biopolítica como parte esencial de la producción del espacio urbano, en donde el control social forma parte de los dispositivos de estabilidad del modelo de acumulación de capital (Hidalgo, et. al., 2017).

El artículo está organizado en cinco secciones. Se discuten las características del concepto de gubernamentabilidad, desarrollado por Foucault, para relacionarlo con la política de vivienda como el mecanismo por el cual se "regula" la vida en sociedad. En la tercera sección, se retoman planteamientos esenciales de la deuda, como un factor central en la actividad inmobiliaria. $\mathrm{Y}$ en la cuarta sección, se analiza la conformación de la Ciudad de México como parte de un espacio producido para la reproducción del capital y exclusión de población de escasos recursos económicos.

Más allá de presentar conclusiones, se ofrecen reflexiones en torno a discutir el ejercicio de poder como una forma de control y gestión de la sociedad a través de las políticas públicas. En el tema de la vivienda, esto puede introducir interrogantes sobre las distintas problemáticas que actualmente se han generado debido a la construcción masiva de vivienda en las periferias urbanas.

\section{Metodología}

Se realizó una revisión bibliográfica que permitiera aproximarnos a conceptos como gubernamentabilidad y biopolítica a partir de los cuales proponemos reflexionar la política de vivienda implementada en México desde el año 2000, como una forma de control, destacando el confinamiento de población de escasos recursos en la periferia urbana de la Ciudad de México. Para lo cual se retomaron diversos trabajos de Michael Foucault, así como de distintos académicos que han discutido sobre el

${ }^{1}$ Se entenderá por Ciudad de México a las 16 alcaldías y los 60 municipios (del estado de México y del estado de Hidalgo) que conforman la llamada Zona Metropolitana del Valle de México. 
tema, y algunos más que han utilizado dichos conceptos para explicar distintos fenómenos.

El confinamiento ha sido posible en la medida que se fortaleció un sistema de crédito para adquisición de vivienda por parte del gobierno federal, razón por la cual, fue necesario obtener información sobre los créditos otorgados por instituciones públicas, de manera particular por el Instituto del Fondo Nacional para la Vivienda de los Trabajadores (Infonavit), el cual es la institución más importante en cuanto a la cantidad de créditos otorgados para la compra de vivienda en México. Para el caso de la Ciudad de México, además de información del Infonavit, se trabajó con información del Fondo de la Vivienda del Instituto de Seguridad y Servicios Sociales de los Trabajadores del Estado (Fovissste), la segunda institución más importante en otorgamiento de créditos. Esta revisión consistió en la consulta de financiamientos otorgados por las instituciones públicas desde el año 2000 hasta el año 2015, la cual está desglosada a nivel municipal, lo que permitió diferenciar aquellos créditos ejercidos en las alcaldías de la Ciudad de México y en los municipios de la zona metropolitana. Aunado a lo anterior, también fue posible identificar, según la tipología de vivienda (económica, media, popular, residencial y residencial plus), hacia dónde se dirigían los créditos para la compra de vivienda nueva o usada. Toda esta información se obtuvo a través del Sistema Nacional de Información e Indicadores de Vivienda, dependiente de la Comisión Nacional de Vivienda.

El análisis exploratorio de los datos obtenidos, de manera particular, los créditos otorgados según veces salario mínimo por el infonavit (10 millones de créditos)2 desde el año 1975 hasta el año 2016 permitió observar diversos patrones que refuerzan los argumentos planteados, de manera particular, el fortalecimiento del sistema de crédito por parte de las instituciones públicas de vivienda a nivel nacional, y la ejecución de estos en la periferia de la Ciudad de México, son elementos que reproducen la deuda en sectores de población de bajos ingresos quienes adquieren una vivienda en la periferia urbana. Aunado a lo anterior, tanto la participación del

${ }^{2}$ En la Cumbre de Financiamiento a la Vivienda Infonavit 2018, celebrada en la Ciudad de México los días 2 y 3 de mayo se otorgó el crédito número 10 millones, por parte del Presidente Enrique Peña Nieto, destacando financiamiento de las instituciones públicas como privadas, son parte de la importancia de la vivienda como un factor de acumulación de capital (Aalbers, 2017; Shimbo, 2012).

La argumentación principal se construyó a partir del concepto de gubernamentabilidad, analizado en tanto las instituciones, estrategias e instrumentos jurídicos ejercen el poder, de cierta forma encubierta. Siguiendo a Raffestin (2015), quien distingue el ejercicio de poder de dos formas: por un lado, aquella mediante la cual se constituye en actos y decisiones directamente del Estado (la forma más visible es mediante el uso de la fuerza) y, por otro lado, de forma que se esconde tan bien que está presente en todo. Esto permitió analizar la política de vivienda como una estrategia política de reproducción de capital y control de la sociedad.

\section{Gubernamentabilidad y política de vivienda}

Hacia la década de 1970, Foucault (2000) desarrolló algunos planteamientos que giran en torno de lo que llamó biopoder. En un recuento histórico, para explicar el origen del concepto, menciona que hasta el siglo XVIII existió un predominio de mecanismos de tipo disciplinario para el ejercicio de poder, sin embargo, a mediados de ese siglo se puede apreciar el surgimiento de otros mecanismos, los cuales no excluyen las técnicas disciplinarias enfocadas al individuo (cuerpo), sino que las engloba, las integra, las modifica. Las nuevas técnicas desarrolladas para el ejercicio de poder, además de considerar las disciplinarias, son mecanismos dirigidos a la colectividad:

A diferencia de la disciplina, que se dirige al cuerpo, esta nueva técnica de poder no disciplinario se aplica a la vida de los hombres e, incluso, se destina, por así decirlo, no al hombre/cuerpo sino al hombre vivo, al hombre ser viviente; en el límite, si lo prefieren, al hombre/especie. (Foucault, 2000, p. 220).

Los mecanismos que hacen referencia al disciplinamiento del cuerpo, los llamó anatomopolítica:

que 1 de cada tres créditos se ha otorgado en el sexenio 2012 - 2018. (La Gaceta, 2018). 
[C]entrado en el cuerpo como máquina: su educación, el aumento de sus aptitudes, el arrancamiento de sus fuerzas, el crecimiento paralelo de su utilidad y su docilidad, su integración en sistemas de control eficaces y económicos, todo ello quedó asegurado por procedimientos de poder característicos de las disciplinas: anatomopolítica del cuerpo humano. (Foucault, 2007b, p.168)

Mientras que, al control de la especie humana, a esa tecnología de poder (biopoder) le llamó biopolítica:

[C]entrado en el cuerpo-especie, en el cuerpo transido por la mecánica de lo viviente y que sirve de soporte a los procesos biológicos: la proliferación, los nacimientos y la mortalidad, el nivel de salud, la duración de la vida y la longevidad, con todas las condiciones que pueden hacerlos variar; todos esos problemas los toma a su cargo una serie de intervenciones y controles reguladores: una biopolítica de la población. Las disciplinas del cuerpo y las regulaciones de la población constituyen los dos polos alrededor de los [169] cuales se desarrolló la organización del poder sobre la vida. El establecimiento, durante la edad clásica, de esa gran tecnología de doble faz anatómica y biológica, individualizante y especificante, vuelta hacia las realizaciones del cuerpo y atenta a los procesos de la vidacaracteriza un poder cuya más alta función no es ya matar sino invadir la vida enteramente. (Foucault, 2007b, pp.168-169)

En su curso dictado en el Collége de France durante 1978 y 1979 la define como: "el conjunto de mecanismos por medio de los cuales aquello que, en la especie humana, constituyen sus rasgos biológicos fundamentales podrá ser parte de una política, una estrategia política, una estrategia general de poder..." (Foucault, 2014, p. 15).

Foucault pone énfasis sobre procesos de natalidad, mortalidad, morbilidad y longevidad, en otras palabras, en cómo los discursos médicos y religiosos recaen sobre la población (Foucault, 2007a), sin embargo, también destaca (y es aquí lo que podemos retomar para el análisis de la política de vivienda), los "mecanismos más sutiles, más racionales, de seguros, de ahorro individual y colectivo, de seguridad, etcétera" (Foucault, 2000, p. 221), es decir, los mecanismos jurídicos y políticos desarrollados por el Estado. De esta manera, el ejercicio de poder, la biopolítica, se puede entender a partir del concepto de gubernamentabilidad, el cual, se define como:

[E]l conjunto construido por las instituciones, los procedimientos, análisis y reflexiones, los cálculos y las tácticas que permiten ejercer esta forma bien específica, aunque compleja, de poder, que tiene por objetivo principal la población, como forma mayor de la economía política, como instrumento técnico fundamental de los dispositivos de seguridad. (Foucault, 2014, p. 136)

Para Foucault "el Estado moderno nace cuando la gubernamentabilidad se convierte efectivamente en una práctica política calculada y meditada" (Foucault, 2014, p. 193). Es la forma en la cual se construyen mecanismos "sutiles" (como forma de dirigir a la población - biopolítica -, contrario a los mecanismos de la anatomopolítica sobre disciplinamiento), como las políticas públicas, las cuales forman parte de estrategias para el control de la población. Para Huxley (2007) estudiar la "gubernamentalidad" implica no solo un examen de las prácticas y programas que buscan moldear, guiar y gobernar el comportamiento de los demás y el yo, sino también, los "regímenes de la verdad", es decir, objetivos y aspiraciones, las mentalidades y racionalidades entrelazadas en los intentos de dirigir las formas de conducta.

Lazzarato analiza la biopolítica en términos de una forma de gobierno y lo define como una "forma de gobierno de una nueva dinámica de las fuerzas que expresan entre ellas relaciones de poder que el mundo clásico no conocía" (2000, p. 84). Por su parte, Tejeda (2012) establece que la biopolítica se puede entender como la presencia del poder y la política en la regulación de nuestras vidas, de nuestra forma de pensar y de actuar, de comportarnos y de existir. Por su parte, Abad (2018) al analizar la gestión de la movilidad establece el entendimiento de la biopolítica no solo mediante la producción jerárquica y desigual de quién o qué (cuerpos), y por dónde (espacios), se puede mover, sino también de cómo, en qué tiempos, debe o puede hacerlo, siempre centrado en el control y dominación de la población. La biopolítica trabaja sobre poblaciones, dejando actuar la libertad (manipulada) de los individuos (León y Urabayen, 2017). De esta manera, podemos apreciar diversas formas 
de regular la vida en sociedad, siendo una de ellas las políticas públicas implementadas por los Estados.

Las políticas públicas, instrumentalizadas como planes de desarrollo, instituciones, etcétera, cumplen objetivos determinados, que pueden ser interpretados como una forma de control de la sociedad y reproducción del capital, algunos de los cuales se pueden encontrar subsumidos en aspectos culturales. Harvey (2007), establece que los aspectos culturales, los cuales interiorizamos como objetivos de vida, son creados a partir de la implementación de políticas públicas. Por ejemplo, en el tema de vivienda, el anhelo de acceder a una vivienda en propiedad, deseo que se ha desarrollado de manera preponderante en países latinoamericanos, resulta ser un elemento esencial para la reproducción de capital y es una forma de gestionar la vida en sociedad, como se discutirá más adelante.

La política de vivienda que se ha desarrollado en México, de manera particular desde el año 2000, cumple por lo menos dos funciones esenciales; por una parte, es un mecanismo para la reproducción y acumulación de capital para las empresas inmobiliarias del sector privado, por otra parte, cumple una función de "organización" y "control" social. Tanto la reproducción de capital como el control y organización de la población forman parte de la gubernamentabilidad de la que habla Foucault.

\section{Vivienda como mecanismo de reproducción de capital}

Mucho se ha discutido sobre las modificaciones en la política de vivienda. El papel del Estado (Esquivel, 2006; Puebla, 1999), la liberalización del suelo (Olivera, 2015; Salinas, 2009), la financiarización de la vivienda (Cattaneo, 2011; De Mattos, 2016; Rolnik, 2014 y 2017) y, de manera particular, el modelo y consecuencias del subsidio a la demanda (Held, 2000; Janoschka y Salinas, 2017; Rodríguez y Sugranyes, 2004 y 2006; Sugranyes, 2006). Aunado a lo anterior, otras investigaciones giran en torno al Estado como el principal ente en el mercado de vivienda, apoyando tanto la oferta como la demanda (Bourdieu, 2002; Calderón, 2015; Salinas, 2016). No obstante, poco se ha discutido respecto a las condiciones previas desarrolladas por el Estado, para que el mercado de compraventa de vivienda sea un éxito en un país donde gran parte de su población vive en pobreza. El anhelo de la vivienda en propiedad, lejos de ser un aspecto aspiracional, es un objetivo de vida, que se ha desarrollado en México desde mediados del siglo pasado.

Se ha fomentado la concepción de la vivienda no como un medio sino como un fin. No como un medio a través del cual se generan las condiciones para la reproducción social, sino como un fin en sí mismo, como un objeto de deseo, pues la vivienda se conforma como un patrimonio familiar. Este anhelo, se ha convertido en un objetivo de vida, una forma de regulación biopolítica, en la cual, sin darnos cuenta, es un elemento esencial de la vida. Por ello, la política de vivienda se ha enfocado en satisfacer este deseo, no como un satisfactor social, sino como un producto esencial en la reproducción de capital (industria de la construcción), así como para la acumulación por parte del sector privado.

El Estado crea instituciones para apoyar la oferta por parte de las inmobiliarias privadas, por un lado, mediante créditos de segundo piso, así como exenciones fiscales y, por otro lado, mediante el establecimiento de un mercado potencial solvente, mediante el sistema de crédito a los consumidores. De esta forma, los mecanismos para la inversión inmobiliaria, el apoyo a la demanda y el anhelo del acceso a la vivienda en propiedad forman un sistema óptimo para la reproducción y acumulación de capital.

\section{Vivienda como mecanismo de organización y control social}

La espacialización de la política de vivienda la podemos observar en la manera en la cual se está configurando la Ciudad de México. Hacia mediados del siglo pasado la expansión de la ciudad se caracterizó en mayor medida por la proliferación de asentamientos informales. Sin embargo, en las dos últimas décadas, el crecimiento urbano está relacionado a la construcción masiva de vivienda económica (Salinas y Pardo, 2018), mientras las áreas centrales se configuran como zonas revalorizadas por el capital.

El mercado formal de vivienda que está caracterizando la expansión de la periferia urbana se ha centrado en producción de vivienda económica, confinando a sectores de la población de ingresos bajos, habitantes que no tienen otras opciones de localización residencial más que en la periferia lejana, la cual carece de infraestructura y equipamiento básico. El mercado de vivienda se ha preocupado por construir vivienda sin la menor relación 
con el desarrollo urbano, es decir, no importa las condiciones para la reproducción de la vida que se está generando sino únicamente que sean productos redituables económicamente.

La política de vivienda está confinando a población de escasos recursos en la periferia, es una manera formal implementada desde el Estado. Sin embargo, un elemento esencial para esta reproducción de capital y confinamiento en las periferias urbanas es el sistema de crédito, que mediante la deuda está generando un control biopolítico de la población endeudada. Se impone una rutina, casa - trabajo, casa - escuela, etcétera, en donde, para los residentes es difícil escapar de esa rutina, la cual puede analizarse como prácticas de control y regulación de las vidas. Es aquí donde el proceso de la construcción de vivienda, su localización y el sistema de crédito para la adquisición de una vivienda en propiedad, emanada desde la política pública, se entiende como gubernamentabilidad.

\section{Deuda y subsidio a la demanda}

Como se ha mencionado, el aspecto cultural ha sido importante para despertar el interés de acceder a la vivienda en propiedad. Para ello, se han desarrollado los mecanismos pertinentes. A partir de la década de los setenta del siglo pasado, se crean las instituciones públicas de vivienda, siendo uno de sus principales objetivos atender el déficit habitacional, para lo cual, además de encargarse de la producción de la vivienda, un elemento central fue el desarrollo del sistema de crédito. Este sistema, es uno de los mecanismos que perduran bajo la lógica neoliberal.

La generación de la deuda, derivada del sistema de crédito (público o privado) no sólo forma parte del esquema del proceso productivo (producción, distribución, circulación, consumo) necesario para la reproducción de capital, en el cual el Estado garantiza la compra de vivienda. Si no, además, forma parte de una relación de poder. Lazzarato (2013) argumenta que la relación acreedor-deudor expresa una relación de fuerzas entre propietarios (del capital) y no propietarios (del capital).

Dos instituciones públicas fueron creadas en los setenta con el objetivo de atender el déficit de vivienda, el Infonavit y el Fovisste. En sus orígenes, mantenían el control de la producción de vivienda (acceso al suelo, diseño, producción, financiamiento), sin embargo, con las reformas neoliberales implementadas en los años noventa, en mayor medida se convirtieron en instituciones hipotecarias (Janoschka y Salinas, 2017; Salinas, 2016). No obstante, la característica del sistema de crédito ha variado. Hasta inicios de la década de los noventa, los créditos otorgados buscaban satisfacer la demanda de población de menores ingresos, sectores de población que tuvieran ingresos hasta dos veces el salario mínimo. Eran créditos para adquirir una vivienda de interés social. Hacia los noventas, debido a que el Estado presentaba una amplia cartera vencida, por recomendaciones del Banco Mundial (mediante el Programa para el Fomento y Desregulación de la Vivienda), se establece como prioridad reducir la cartera vencida, de manera que los créditos se otorgarían a sectores de población con mayor solvencia económica, es decir, que ganaran más de 2 veces el salario mínimo (Tabla 1). Pero, además, los créditos deberían de obtener beneficios, para lo cual se elevan las tasas de interés (Boils, 2004), al punto de llegar a igualar la tasa de interés ofrecida por la banca comercial, es decir, se cambia la función para la cual estaban originado, ya no se procura la compra de vivienda social, sino únicamente la dinamización del mercado de vivienda. 
Las reformas que se han realizado en el sistema de crédito son acordes a la lógica de mercado, reconociendo la deuda como un factor esencial de crecimiento: la deuda

constituye (...) el motor económico y subjetivo de la economía contemporánea. La fabricación de deudas, es decir, la construcción y el desarrollo de la relación de poder entre acreedores y deudores, se ha pensado y programado como el núcleo estratégico de las políticas neoliberales. (Lazzarato, 2013, p. 30)

De acuerdo con el planteamiento anterior, el mecanismo de crédito para la adquisición de vivienda no es sólo un elemento importante para el mantenimiento del mercado (compraventa de vivienda), sino, además, forma parte del control sobre la sociedad.

En un interesante estudio Lazzarato (2013) retoma la teoría nietzscheana del crédito y la teoría marxista de la moneda, por cuanto invitan a "una lectura no economicista de la economía, en cuyo fundamento no se haya el intercambio, sino una relación de poder asimétrico entre acreedor y deudor" (Lazzarato, 2013, p. 83). Una lectura no economicista de la economía significa que la producción económica es indisociable de la producción y el control de la subjetividad y sus formas de existencia. No basta con analizar el peso en la actividad económica de la industria de la construcción, las inversiones por parte del sector inmobiliario, la inversión pública mediante los Organismos Nacionales de Vivienda ${ }^{3}$ (ONAVIS) en el sistema de crédito, sino además, resulta interesante discutir cómo se interrelacionan con aspectos subjetivos, tales como el anhelo de la vivienda en propiedad, la expulsión y concentración de sectores de la población quienes no tienen ningún derecho de decidir sobre la localización de las viviendas. Es el mercado quien decide la localización y las condiciones de la vivienda (Salinas y Pardo, 2018), mientras que, para ello, se han entretejidos mecanismos de control social, emanados de políticas públicas.
Pensar la deuda más allá de lo estrictamente económico, del compromiso adquirido sobre un acreedor, sino como una forma de control de la sociedad:

[E]l usuario transformado en "deudor" no tiene que hacer sus rembolsos en dinero constante $y$ sonante, sino en comportamientos, actitudes, maneras de actuar, proyectos, compromisos subjetivos, tiempo dedicado a la búsqueda de empleo, tiempo utilizado para formarse de acuerdo con los criterios dictados por el mercado y la empresa, etc. La deuda remite directamente a una disciplina de vida y a un estilo de vida que implica un trabajo sobre "sí mismo", una negociación permanente consigo mismo, una producción de subjetividad específica: la del hombre endeudado. En ese sentido, puede firmarse, sin duda, que la deuda reconfigura el poder biopolítico, al implicar una producción de subjetividad propia del hombre endeudado. (Lazzarato, 2013, pp. 120-121).

Por lo anterior, el control social que se aprecia a partir de la implementación de la política de vivienda no es únicamente en confinar a población de escasos recursos en las periferias urbanas sino una forma de incidir en sus vidas mediante "ataduras" generadas por esta relación de poder, deudor-acreedor, en el que el Estado es uno de los actores principales.

\section{Configuración reciente de la Ciudad de México}

La política de vivienda, analizada como un mecanismo de la gubernamentabilidad, ha permitido el control social y la reproducción del capital, se puede apreciar en la configuración de la Ciudad de México. Desde el año 2000 y durante los siguientes dos sexenios se mantuvo una política para favorecer la expansión urbana a partir de la construcción masiva de vivienda, dando como resultado la construcción de más de 600.000 viviendas, de las cuales el $92 \%$ es vivienda económica. Esto supone, según las estimaciones de la Secretaría de Desarrollo Urbano y Metropolitano del Gobierno del Estado de México, la

estatal y municipal, no obstante, las más destacadas por el número de créditos como por el monto de estos son las instituciones señaladas Infonavit y Fovissste.
3 Los organismos nacionales de vivienda son aquellas instituciones
públicas que otorgan créditos para la adquisición, construcción y
reparación de vivienda. Operan en los tres niveles de gobierno, federal, 
concentración de poco más de 2.400 .000 nuevos residentes.

En los municipios de la zona metropolitana, en particular al norte de la periferia, se concentró más del $50 \%$ de la construcción de vivienda en tan sólo cuatro municipios (Huehuetoca, Zumpango, Tecámac y Tizayuca), los cuales se localizan entre 50 y $60 \mathrm{~km}$ de distancia de la zona central de la ciudad. La construcción masiva de vivienda se realizó en conjuntos habitacionales, los cuales pueden contener desde 4.000 a más de 20.000 viviendas (Figura 1). La localización de estos conjuntos representa un problema debido a la carencia de empleo, educación y servicios médicos de buena calidad, así como insuficiente servicio de transporte y deficiente equipamiento e infraestructura urbana. La construcción masiva de vivienda forma parte del confinamiento de población de bajos ingresos en la periferia urbana.

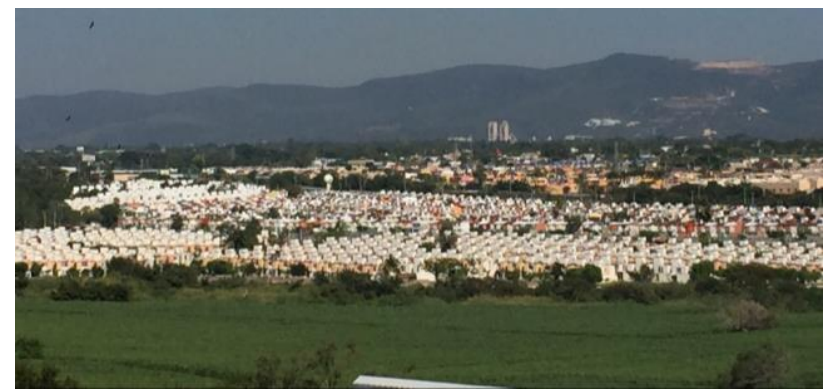

Figura 1. Conjunto habitacional en la periferia urbana. Fuente: Fotografía del autor, 2016.

En el periodo mencionado se otorgaron la mayor cantidad de créditos por parte de organismos públicos, destacando de manera particular los que se ejercieron para comprar vivienda en los municipios de la zona metropolitana. Como se puede observar en la Tabla 2 , del año 2001 al 2015 poco más del 70\% de los créditos para adquirir vivienda en la Ciudad de México se ejercieron en los municipios periféricos.

Tabla 2

Créditos otorgados por Infonavit y Fovisste, 2000 - 2015

\begin{tabular}{cccc}
\hline & $\begin{array}{c}\text { Ciudad de } \\
\text { México }\end{array}$ & $\begin{array}{c}\text { Municipios de } \\
\text { la ZMVM }\end{array}$ & ZMVM \\
\hline $2000-2005$ & 60,935 & 153021 & 213,956 \\
$2006-2010$ & 67,474 & 248,001 & 315,475 \\
$2011-2015$ & 152,106 & 267,397 & 419,503 \\
$2001-2015$ & 280,515 & 668,419 & 948,934 \\
\hline
\end{tabular}

Fuente: Elaboración propia con información del Sistema Nacional de Información e Indicadores de Vivienda (Comisión Nacional de Vivienda, s.f.).

No obstante, la política de vivienda fue modificada durante el sexenio 2012-2018, y es mediante el Plan Nacional de Vivienda (2013-2018) que se busca detener la expansión urbana, y ahora, el objetivo es regresar a la ciudad construida. A diferencia del modelo anterior en el cual bastaba con un crédito proveniente de instituciones públicas, éstos no son suficientes para adquirir vivienda en áreas centrales, razón por la cual, son los bancos quienes otorgan créditos a sectores de población de ingresos medios y altos. Como se puede apreciar en la Tabla 3 , los créditos otorgados por instituciones financieras privadas de 2010 a 2015, para las alcaldías centrales de la Ciudad de México, se concentran en vivienda media, residencial y residencial plus. Por su parte, la mayor cantidad de créditos otorgados por los ONAVIS en los municipios que forman parte de la zona metropolitana se concentran en vivienda económica, popular y tradicional. 


\section{Tabla 3}

Acciones de entidades financieras y ONAVIS para la ZMVM, 2010-2015

\begin{tabular}{|c|c|c|c|c|c|c|}
\hline & \multicolumn{6}{|c|}{ Acciones* de entidades financieras 2010 - 2015} \\
\hline & Económica & Media & Popular & Residencial & $\begin{array}{c}\text { Residencial } \\
\text { Plus }\end{array}$ & Tradicional \\
\hline $\begin{array}{c}\text { Municipios de la } \\
\text { ZMVM }\end{array}$ & 260 & 9271 & 2584 & 5493 & 3379 & 8824 \\
\hline $\begin{array}{c}\text { Alcaldías de la } \\
\text { Ciudad de México }\end{array}$ & 249 & 19584 & 2523 & 13680 & 7597 & 7839 \\
\hline \multirow[t]{3}{*}{ Total ZMVM } & 509 & 28855 & 5107 & 19173 & 10976 & 16663 \\
\hline & \multicolumn{6}{|c|}{ Acciones de ONAVIS 2010 - 2015} \\
\hline & Económica & Media & Popular & Residencial & $\begin{array}{c}\text { Residencial } \\
\text { Plus }\end{array}$ & Tradicional \\
\hline $\begin{array}{l}\text { Municipios de la } \\
\text { ZMVM }\end{array}$ & 562 & 18956 & 78874 & 6476 & 1170 & 84204 \\
\hline $\begin{array}{c}\text { Alcaldías de la } \\
\text { Ciudad de México }\end{array}$ & 26 & 33113 & 1498 & 15833 & 2971 & 16089 \\
\hline Total ZMVM & 588 & 52069 & 80372 & 22309 & 4141 & 100293 \\
\hline
\end{tabular}

Fuente: elaboración propia con información del Sistema Nacional de Información e Indicadores de Vivienda (Comisión Nacional de Vivienda, S.f.).

* Por acciones se entiende los créditos otorgados para la adquisición de vivienda nueva o usada.

Si bien es cierto, el contexto histórico de la conformación de la Ciudad de México no permite establecer una clara diferenciación entre la "periferia pobre" y las "áreas centrales ricas". No se puede negar que hay una marcada mezcla en la tipología de vivienda, es decir, en áreas centrales existe población de escasos recursos así como en la periferia urbana. De la misma manera, en zonas centrales vive población de ingresos medios y altos así como en determinadas zonas suburbanas (no en la periferia). Lo importante a destacar, es una clara tendencia, desarrollada desde la política pública, para excluir de la dinámica del mercado formal de vivienda a sectores de población de bajos ingresos de las áreas centrales, pues no hay (con escasas excepciones como las acciones implementadas por el Instituto de Vivienda de la Ciudad de México), construcción de vivienda económica.

Mientras que la periferia, independientemente de las condiciones generadas (infraestructura y equipamiento urbano), se destinó para sectores de bajos recursos. Es el mecanismo de control de la sociedad:

[L]a ciudad no es pensada para el sujeto, pues se homogeneiza un proyecto de planificación urbana y de administración de las conductas que desconoce la subjetividad como construcción ética y política de pensar y concebir un espacio para el encuentro y la discusión humana, incluso para la contemplación, la reflexión y la acción estética, al primar lugares de disciplinamiento donde solo viven el asfalto, la carne y la piedra (Sennett, 1997), haciendo de las diferencias lugares de domesticación biopolítica (...). (Cardona y Cardona, 2016, p. 87)

Esto ha traído distintas problemáticas para los residentes que han hecho realidad su deseo de adquirir una vivienda en propiedad, sin considerar las distintas adversidades a las que se enfrentan, tales como: carencia de servicios básicos, lejanía al empleo y servicios como educación y salud, inseguridad, entre otros, lo que origina, incluso, en el abandono de vivienda (Figura 2), en donde tal y como menciona Sennett (1997) solo viven el asfalto, la carne y la piedra ( Figura 3 ). 


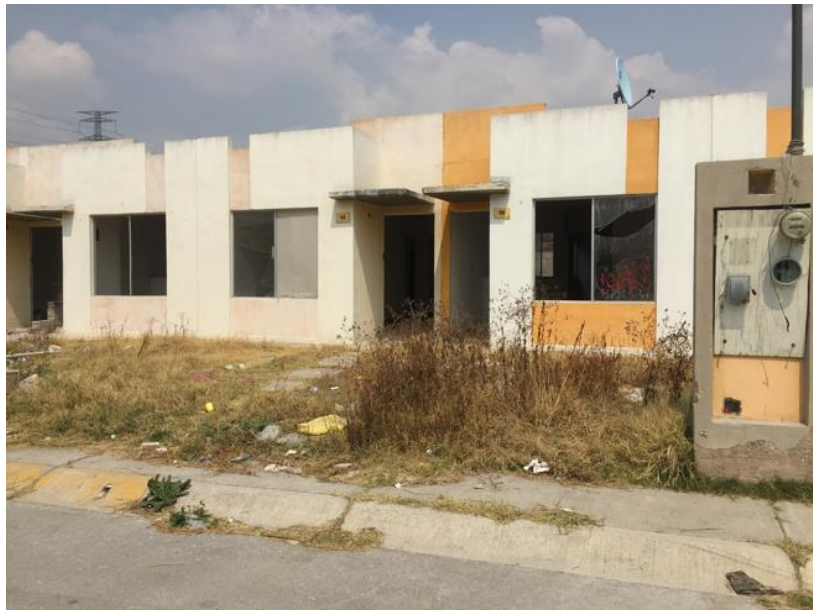

Figura 2. Vivienda abandonada en la periferia urbana. Fuente: Fotografía del autor, 2016.

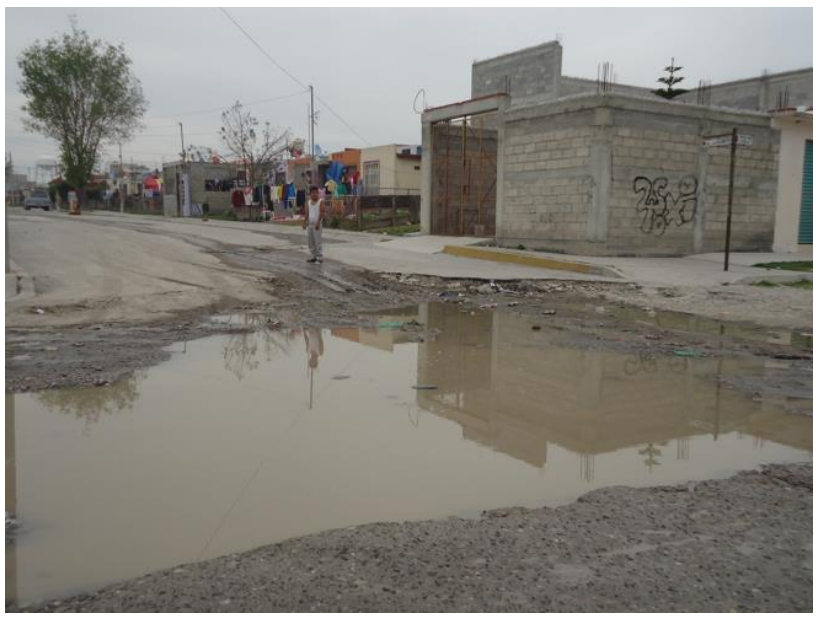

Figura 3. Vivienda para población de bajos ingresos. Fuente: Fotografía del autor, 2016.

Se ha mencionado que la construcción masiva de vivienda económica en la periferia urbana ha contribuido a la reproducción de capital y al control de la sociedad, pero es necesario destacar, que la implementación de estas políticas, dentro de la dinámica de la producción del espacio (Lefebvre, 2013) está sujeta a la mediación de las condiciones del espacio preexistente, es decir, no se reproducen en un espacio abstracto. La configuración de la periferia de la Ciudad de México presenta condiciones generales derivadas de la política de vivienda, sin embargo, sus características propias, resistencias, aspectos político-institucionales y culturales determinarán diversos arreglos que pueden analizarse en el espacio producido. Justamente es una de las discusiones sobre el abordaje del espacio absoluto kantiano abordado por Foucault (Harvey, 2013; Thrift, 2013). Por tanto, es importante considerar las dimensiones desde el surgimiento mismo de la política de vivienda así como su implementación.

\section{Conclusiones}

Uno de los factores esenciales del ejercicio de poder (biopolítica) desarrollado como forma de gubernamentabilidad, es que no se basa en una intervención directa, un tipo de disciplinamiento, tal como se realizaba en sociedades medievales, sino bajo condiciones de "libertad". En el capitalismo, y de manera particular, en el modelo neoliberal, la libertad y la propiedad privada son los pilares en los que se sustenta dicho modelo (Harvey, 2007). En esta libertad, la biopolítica penetra de manera "indirecta" en la sociedad. Aquí se intentó retomar el caso de la política de vivienda, como una forma de acumular capital a partir de la producción masiva de vivienda social en la periferia y también como una forma de ejercer el control de la sociedad.

La construcción cultural del patrimonio, así como la imperiosa necesidad de la propiedad privada han jugado un rol muy importante en la dinámica del mercado formal de vivienda. Se ha convertido en un ideal muy arraigado en el imaginario colectivo, impidiendo una mayor discusión que permita otras formas de acceso a la vivienda que no sea únicamente mediante la propiedad privada. El mercado inmobiliario ha logrado posicionar a la vivienda no como un satisfactor social, sino como un fin de carácter económico, en otras palabras, destacando el valor de cambio sobre el valor de uso.

Como forma de controlar a la población se puede designar espacios para la producción masiva de vivienda para población de escasos recursos, esto es, una estrategia de concentrar a la población en las periferias urbanas. Otra forma de control es aquel desarrollado mediante el mecanismo de la deuda, en el cual hay una modificación en la vida misma, en el comportamiento, formas de actuar, etcétera, todo ello, teniendo como uno de los principales actores al Estado junto con la banca comercial.

Estas dos dimensiones que se han desarrollado a partir de la construcción masiva de vivienda en la periferia de la Ciudad de México han determinado y a su vez están determinadas por las características del espacio construido. Lo que se pretende resaltar es que la lógica de 
la expansión de la periferia resulta más compleja que el simple hecho de pensar planificar el territorio (entendido como una dimensión física) e implementar una política pública.

El biopoder ha sido un elemento indispensable en el desarrollo del capitalismo (Foucault, 2007b), pero incluso, la instrumentalización de la gubernamentabilidad, como se vio con el tema de la política de vivienda, ya no se desprenden únicamente desde la administración pública sino también desde las entidades no estatales, a lo que se le ha denominado "gubernamentabilidad neoliberal" (Ferguson y Gupta, 2002) [B]

\section{Referencias}

Abad, B. (2018). Regímenes de movilidad y expropiación del tiempo: la espera como cronopolítica. Arbor, 194(788), 1-13.

Aalbers, M. B. (2017). The Variegated Financialization of Housing. International Journal of Urban and Regional Research, 41(4), 542-554. https://doi.org/10.1111/1468-2427.12522

Boils, G. (2004). El Banco Mundial y la política de vivienda en México. Revista Mexicana de Sociología, 66(2), 345367.

Bourdieu, P. (2002). Las estructuras sociales de la economía. Buenos Aires: Manantial.

Calderón, J. (2015). Programas de vivienda social nueva y mercados de suelo urbano en el Perú. EURE (Santiago), (41)122, 27-47. https://doi.org/10.4067/s025071612015000100002

Cardona, H y Cardona, J. (2016). Heterotopías urbanas. Espacios de la exclusión y miradas biopolíticas de Medellín en el siglo XX. Ciencias Sociales y Educación, 5(9), 81-104. https://doi.org/10.22395/csye.v5n9a4

Cattaneo, R. (2011). Los fondos de inversión inmobiliaria y la producción privada de vivienda en Santiago de Chile: ¿Un nuevo paso hacia la financiarización de la ciudad? EURE (Santiago), 37(112), 5-22. https://doi.org/10.4067/s0250-71612011000300001

De Mattos, C. (2016). Financiarización, valorización inmobiliaria del capital y mercantilización de la metamorfosis urbana. Sociologias, 18(42), 24-52. https://doi.org/10.1590/15174522-018004202
Esquivel, M. (2006). Política habitacional y calidad de vida: impacto de los nuevos desarrollos habitacionales. En Borjas y Bucio (Coord.) La vivienda en México. Construyendo análisis y propuestas (pp. 83-104). México: Centro de Estudios Sociales y de Opinión Pública Cámara de Diputados / LIX Legislatura.

Ferguson, J. y Gupta, A. (2002). Spatializing States: Toward an Ethnography of Neoliberal Governmentality. American Ethnologist, 29(4), 981-1002. https://doi.org/10.1525/ae.2002.29.4.981

Foucault, M. (2000). Defender la sociedad. Argentina: Fondo de Cultura Económica.

Foucault, M. (2007a). Nacimiento de la biopolítica. Argentina: Fondo de Cultura Económica.

Foucault, M. (2007b). Historia de la sexualidad 1. La voluntad de saber. México: Siglo XXI editores.

Foucault, M. (2014). Seguridad, territorio, población. México: Fondo de Cultura Económica.

Huxley, M. (2007) Geographies of Governmentality. En Jeremy Crampton \& Stuart Elden (Eds.) Space, Knowledge and Power. Foucault and Geography, England: Ashgate Publishing Limited.

Harvey, D. (2007). Breve historia del Neoliberalismo. España: AKAL.

Harvey, D. (2013) The Kantian Roots of Foucault's Dilemmas. En Jeremy Crampton \& Stuart Elden (Ed.) Space, Knowledge and Power. Foucault and Geography. England: Ashgate Publishing Limited.

Held, G. (2000). Políticas de viviendas de interés social orientadas al mercado: experiencias recientes con subsidios a la demanda en Chile, Costa Rica y Colombia. Santiago de Chile: CEPAL. 
Instituto del Fondo Nacional de la Vivienda para los Trabajadores. Informe anual de actividades. (2015). Disponible en https://portalmx.infonavit.org.mx/wps/wcm/connect 3306ce250-8949-4e65-a59c-

2f54d4dc19ad/Informe Anual de Actividades 2015. pdf?MOD=AJPERES\&CVID $=m d g 83 X_{0}$

Instituto del Fondo Nacional de la Vivienda para los Trabajadores. Informe anual de actividades 2016. Disponible en https://portalmx.infonavit.org.mx/wps/wcm/connect 107e5ff2b-e316-4f07-b94b-

df7f7e742b3e/Informe anual de actividades 2016.p df?MOD=AJPERES\&CVID=mdg8fhO

Instituto del Fondo Nacional de la Vivienda para los Trabajadores. Solicitud de información mediante la Ley Federal de Transparencia y Acceso a la Información Pública.

Kosik, K. (1967). Dialéctica de lo Concreto. México: Editorial Grijalbo.

Dattwyler, R. H., Peterson, V. C. A., y Rivas, D. S. (2017). La espacialidad neoliberal de la producción de vivienda social en las áreas metropolitanas de Valparaíso y Santiago (1990-2014): ¿hacia la construcción idelógica de un rostro humano? Cadernos Metrópole, 19(39), 513-535. https://doi.org/10.1590/2236-9996.2017$\underline{3907}$

Janoschka, M. y Salinas, L. (2017). Peripheral urbanisation in Mexico City A comparative analysis of the uneven social and material geographies in low-income housing estates. Habitat International. A Journal for the Study of Human Settlements, (70), 43-49. https://doi.org/10.1016/i.habitatint.2017.10.003

El Infonavit entrega siu crédito 10 millones en su historia (2018). La Gaceta, 9(50). Disponible en http://portal.infonavit.org.mx/wps/wcm/connect/e2c 815ff-7242-4306-aa7c6c039dc6de04/LaGaceta_50.pdf?MOD=AJPERES\&CO NVERT TO=url\&CACHEID=ROOTWORKSPACEe2c815ff-7242-4306-aa7c-6c039dc6de04-mfgYJH-

Lazzarato, M. (2013). La fábrica del hombre endeudado. Argentina: Amorrortu editores.
Lazzarato, M. (2000). Del biopoder a la biopolítica. Multitudes, (1). Disponible en https://marceloexposito.net/pdf/trad lazzarato biop oderbiopolitica.pdf

Lefebvre, H. (2013). La producción del espacio. España: Capitan Swing.

León, C. J. y Urabayen, J. (2017). Heterotopía y capitalismo en arquitectura. La función ideológica de las Heterotopías como discurso propio de la disciplina arquitectónica en la era de la gobernanza biopolítica. Arbor, 193(784), 1-13. https://doi.org/10.3989/arbor.2017.784n2008

Olivera, G. (2015). La incorporación de suelo social al crecimiento urbano de Cuernavaca y sus efectos en el desarrollo urbano formal e informal del suelo y la vivienda. En Olivera, G. (coord.) La urbanización social y privada del ejido: ensayo sobre la dualidad del desarrollo urbano en México (pp. 149-196). México: CRIM, UNAM,

Puebla, C. (1999). Del Estado interventor al Estado facilitador. Ciudades, (44).

Puebla, C. (2006). El Instituto del Fondo Nacional de la Vivienda para los Trabajadores (Infonavit), en: René Coulomb y Martha Schteingart (coord.) Entre el Estado y el mercado: la vivienda en el México de hoy, Universidad Autonoma Metropolitana Azcapotzalco. México: Miguel Ángel Porrúa.

Raffestin, C. (2015). Por una geografía del poder. México: El colegio de Michoacán.

Rodríguez, A. y Sugranyes, A. (2004). El problema de vivienda de los "con techo". EURE (Santiago), 30(91), 53-65. https://doi.org/10.4067/s025071612004009100004

Rodríguez, A. y Sugranyes, A. (2006). El problema de vivienda de los "con techo". En Alfredo Rodríguez y Ana Sugranyes (Eds.) Los con techo. Un desafío para la política de vivienda social (pp. 59-80). Chile: Ediciones, SUR.

Rolnik, R. (2014). Neoliberalismo reciente: la financialización de la vivienda propia y el derecho a la vivienda. Revista Bitácora, Urbano Territorial, 24(1) 95-104. 
Rolnik, R. (2017). La guerra de los lugares. La colonización de la tierra y la vivienda en la era de las finanzas. Chile: Ediciones LOM.

Salinas, L. (2009). El Estado en el proceso de acumulación por desposesión en el Municipio de Chalco, Estado de México. Cuadernos de Geografía, Revista Colombiana de Geografía, n. ${ }^{\circ} 18$, pp. 25-34.

Salinas, L. (2016). Política de vivienda y gestión metropolitana en la expansión de la periferia de la ZMCM. Cuadernos Geográficos, 55(2), 217-237.

Salinas, L. y Pardo, A. (2018). Urbanismo neoliberal en la expansión reciente de la Ciudad de México. Revista Bitácora, Urbano Territorial, 1, 117-123.

Sennett, R. (1997). Carne y piedra: el cuerpo y la ciudad en la civilización occidental. Madrid: Alianza.

Shimbo, L. (2012). Vivienda social de mercado: confluencia entre Estado, empresas constructoras y capital financiero. México: Infonavit, UAEMEX, Facultad de Ciencias Políticas y Sociales.

Comisión Nacional de Vivienda (s.f.). Sistema Nacional de Información e Indicadores de Vivienda. Disponible en http://sniiv.conavi.gob.mx:9000/inicio/index.aspx

Sugranyes, A. (2006). La política habitacional en Chile, 1980-2000: un éxito liberal para dar techo a los pobres. En Alfredo Rodríguez y Ana Sugranyes (Eds.), Los con techo (pp. 25-59). Un desafío para la política de vivienda social. Chile: Ediciones SUR.

Tejeda, J. L. (2012). Biopolítica, control y dominación. Espiral, Estudios sobre Estado y Sociedad, 18(52), $77-$ 107.

Thrift, N. (2013). Overcome by Space: Reworking Foucault. En Jeremy Crampton \& Stuart Elden (Eds) Space, Knowledge and Power. Foucault and Geography, England: Ashgate Publishing Limited. 